\section{FRI0327 RELIABILITY OF LA AND DIAGNOSTIC PERFORMANCE OF APS/PT IN DIFFERENT CLINICAL SETTINGS OF APS: A MULTICENTER STUDY}

M. Radin ${ }^{1}$, I. Cecchi ${ }^{1}$, E. Rubini ${ }^{1}$, A.M. Scotta ${ }^{1}$, R. Rolla ${ }^{2}$, B. Montaruli ${ }^{3}$, P. Pergolini ${ }^{2}$, G. Mengozzi ${ }^{1}$, E. Muccini ${ }^{1}$, D. Roccatello ${ }^{1}$, S. Sciascia ${ }^{1} .{ }^{1}$ Università Degli Studi Di Torino, Turin; ${ }^{2}$ Università del Piemonte Orientale, Novara; ${ }^{3}$ Ospedale Mauriziano, Turin, Italy

Background: Correct interpretation of lupus anticoagulant (LA) tests is crucial for diagnosis of antiphospholipid syndrome (APS). However, testing patients during vitamin $\mathrm{K}$ antagonist (VKA) or other anticoagulant remains a contentious issue and has been discouraged by official guidelines ${ }^{1}$ because of interpretational problems affecting the mixing test. Similarly, the clinical significance of weak LA, especially in the context of VKA, remains uncertain and certainly needs a more thorough evaluation. Autoantibodies that recognise a phosphatidylserine/prothrombin (PS/PT) complex have been reported to be associated with APSandmay have diagnostic relevance in these settings.

Objectives: To evaluate the reproducibility of LA testing when performed in different centres and to assess the diagnostic performance of anti-PS/PT in different clinical settings of APS.

Methods: aPL testing was performed in a blind fashion in 4 centres. LA was tested as per the current criteria from the ISTH Subcommittee on LA-Phospholipid-dependent antibodies. ${ }^{1}$ Thirty-two patients were enrolled in this study, as follows: 13 patients with thrombotic APS treated with vitamin $\mathrm{K}$ antagonist; 5 patients thrombotic APS treated with DOAC; 14 patients with transitory/low aPL titer. Anti$\mathrm{PS} / \mathrm{PT}$ IgG/IgM (aPS/PT, Inova Diagnostics)were tested by ELISA. We analysed the categorical agreement and degree of linear association, for LA and aPS/PT, respectively.

Results: Demographic, clinical and laboratory characteristics are summarised in table 1. Categorical agreement for LA among the centres, as expressed by Cohen's kappa coefficients, ranged between 0.61 and 0.80 (as substantial agreement). The correlation among quantitative results in the aPS/PT IgG was strong (when dichotomizing for positive Vs. negative results, Cohen's kappa coefficients $=0.81-1$. Spearman rho 0.84$)$. We observed $12(37.5 \%$ of the total) cases $(7 / 12,58 \%$ patients on VKA) in which LA results were discordant(as defined by lack of agreement in $\geq 2$ laboratories) or inconclusive. Conversely, in those cases, we observed a good correlation for aPS/PT IgG testing (Cohen's kappa coefficients $=0.81-1$, Spearman rho 0.86).

\begin{tabular}{|c|c|c|}
\hline & Patients & $\%$ (32 Patients) \\
\hline \multicolumn{3}{|l|}{ Anagraphic } \\
\hline mean age (S.D.) at data collection & $49,4(14,1)$ & \\
\hline gender(females) & 20 & 62.5 \\
\hline \multicolumn{3}{|l|}{ Clinical manifestations of APS } \\
\hline Thrombosis & 25 & 81.3 \\
\hline Arterial Thrombosis & 10 & 31.3 \\
\hline Venous Thrombosis & 15 & 46.9 \\
\hline Obstetric APS & 0 & 0.0 \\
\hline \multicolumn{3}{|l|}{ aPL profile at diagnosis } \\
\hline LA (positive, $n$ ) & 26 & 81.3 \\
\hline ACA $(\lg G / M)$ & 26 & 81.3 \\
\hline 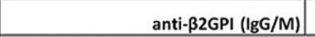 & 21 & 65.6 \\
\hline \multicolumn{3}{|l|}{ Anticoagulant Therapy } \\
\hline VKA (warfarin) & 13 & 40.6 \\
\hline LMWH & 3 & 9.4 \\
\hline DOAC & 5 & 15.6 \\
\hline None & 11 & 34.4 \\
\hline
\end{tabular}

Conclusions: Despite the progress in the standardisation of aPL testing, we observed up to $37.5 \%$ of discrepant results for LA, especially in patients on VKA. Our findings showed that some discordances in the reliability of LA testing still exist. The introduction of aPS/PT antibodies in the diagnostic process of APS might represent a further diagnostic tool, especially when LA is not available or nor reliable.

\section{REFERENCE:}

[1] Pengo V, et al. Update of the guidelines for lupus anticoagulant detection. Subcommittee on Lupus Anticoagulant/Antiphospholipid Antibody of the Scientific and Standardisation Committee of the International Society on Thrombosis and Haemostasis. J Thromb Haemost 2009;7:1737-40.

\section{FRI0328 TARGETED THERAPY USING INTRADERMAL INJECTION OF ETANERCEPT FOR REMISSION INDUCTION IN DISCOID LUPUS ERYTHEMATOSUS (TARGET-DLE): FIRST RESULTS FROM A PROOF-OF- CONCEPT PHASE 2 TRIAL}

M.Y. Md Yusof ${ }^{1}$, M. Wittmann ${ }^{1}$, C. Fernandez ${ }^{2}$, D. Wilson ${ }^{2}$, S. Edward ${ }^{3}$, G. Abignano ${ }^{1}$, A. Alase ${ }^{1}$, L. Sharples ${ }^{2}$, P. Laws ${ }^{4}$, M.J. Goodfield ${ }^{4}$, E.M. Vital ${ }^{1}$ P. Emery ${ }^{1}{ }^{1}$ Leeds Institute of Rheumatic and Musculoskeletal Medicine; ${ }^{2}$ Leeds Institute of Clinical Trials Research, University of Leeds; ${ }^{3}$ Department of Histopathology; ${ }^{4}$ Department of Dermatology, Leeds Teaching Hospitals NHS Trust, Leeds, UK

Background: A significant proportion of patients with discoid lupus erythematosus (DLE) are resistant to conventional therapies. Tumour necrosis factor (TNF) is pathogenic in DLE. A concern with systemic TNF-i administration is induction of pathogenic autoantibodies and flare of disease. This could be overcome using a low-dose intra-dermal injection, which may be sufficient to neutralise the TNF in lesions, without systemic TNF effects.

Objectives: To assess the efficacy and safety of a novel route of administration of a TNF-i using a low dose intra-dermal injection of etanercept (ETN) for remission induction in DLE.

Methods: A prospective single arm, Simon's 2-stage minimax design with Hybrid adaptation, phase II open label trial was conducted in Leeds [NCT02656082]. Key inclusion criteria were i) adults aged $18-80 \mathrm{y}$; ii) $\geq$ one active DLE lesion and iii) refractory to anti-malarials. One index lesion with the highest activity was treated with weekly intra-dermal injection of up to $10 \mathrm{mg}$ ETN. The primary endpoint was $\geq 6$ patients achieving the modified limited Score of Activity and Damage in DLE (ML-SADDLE) 20 response (defined as reduction $\geq 20 \%$ in total activity comprises erythema, induration and scaling from baseline) at Week 12 for a Phase 3 trial to be recommended. Secondary endpoints included change in objective outcome measures; lesional thermography and laser Doppler imaging.

Results: All 25 DLE patients were recruited over 18 months (18 female, mean age $47 \pm 12$ y, 6 had SLE, 9 had positive ANA and median (range) no. of previous systemic therapies was $5(1-16) 17$ patients completed the primary efficacy assessment [Did not attend Week 12 visit $=1$, early withdrawals $=7$ (personal choice $=2, \mathrm{AE}=2$, worsening of $\mathrm{DLE}=1$, non-compliance $=1$, pregnant=1)]. The primary endpoint was met with $13 / 25(52 \%, 95 \% \mathrm{Cl} 31-73)$ meeting the ML-SADDLE 20 in full-set analysis. The rates for ML-SADDLE 50 and 70 were $48 \%$ and $20 \%$ respectively. Key secondary endpoints were met (table 1). Fifty-one AEs (treatment-emergent=28, Grade $3 / 4=4$ ) were recorded. There was no worsening of BILAG or SLEDAI in patients with SLE. Trough serum ETN levels were detected in $6 / 23(26 \%)$.

Abstract FRI0328 - Table 1. Secondary endpoints (per protocol; $n=17$ )

\begin{tabular}{lccc}
\hline Endpoint & $\begin{array}{c}\text { Pre- } \\
\text { Treatment }\end{array}$ & $\begin{array}{c}\text { Post- } \\
\text { Treatment }\end{array}$ & $\begin{array}{c}\text { p- } \\
\text { value }\end{array}$ \\
\hline Physician VAS, mean (SD) mm & $53.1(16)$ & $23.2(20)$ & $<0.001$ \\
Patient VAS, mean (SD) mm & $56.9(28)$ & $29.7(28)$ & 0.001 \\
DLQI, mean (SD) & $11.4(7)$ & $6.5(6)$ & $<0.001$ \\
Laser Doppler Imaging, mean (SD) perfusion & $495.1(224)$ & $376.2(223)$ & 0.018 \\
unit & & & \\
Infrared thermography, mean (SD), ${ }^{\circ}$ Celcius & $1.92(1.17)$ & $1.08(1.05)$ & 0.005 \\
\hline
\end{tabular}

Conclusions: Intradermal injection of ETN substantially reduced clinical activity, met its primary, secondary endpoints and was tolerable in DLE patients who were refractory to anti-malarials and other systemic therapies. This drug warrants further development in multi-centre trials. Analyses of other imaging and histological biomarkers are ongoing and can help stratifying patients for response.

Acknowledgements: This research was funded by NIHR (DRF-2014-07-155) and Pfizer IIR Grant (WI188416). The views expressed are those of the author(s) and not necessarily those of the NHS, NIHR or DOH.

Disclosure of Interest: M. Y. Md Yusof: None declared, M. Wittmann: None declared, C. Fernandez: None declared, D. Wilson: None declared, S. Edward: None declared, G. Abignano: None declared, A. Alase: None declared, L. Sharples: None declared, P. Laws: None declared, M. J. Goodfield: None declared, E. M. Vital: None declared, P. Emery Grant/research support from: Abbott, BMS, Pfizer, MSD and Roche, Consultant for: BMS, Abbott, Pfizer, MSD, Novartis, Roche and UCB

DOI: 10.1136/annrheumdis-2018-eular.6623 


\section{FRI0329 THE NET-EFFECT OF COMBINING RITUXIMAB WITH BELIMUMAB IN SEVERE SYSTEMIC LUPUS ERYTHEMATOSUS}

O. Teng ${ }^{1}$, T. Kraaii ${ }^{1}$, S. Kamerling ${ }^{1}$, E. de Rooy ${ }^{1}$, P. van Daele ${ }^{2}$, O. Bredewold ${ }^{1}$, J. Bakker ${ }^{3}$, I. Bajema ${ }^{4}$, H. Scherer ${ }^{5}$, R. Toes ${ }^{5}$, T. Huizinga ${ }^{5}$, T. Rabelink ${ }^{1}$, C. van Kooten ${ }^{1} .{ }^{1}$ Nephrology, Leiden University Medical Center, Leiden; ${ }^{2}$ Clinical Immunology, Erasmus Medical Center, Rotterdam; ${ }^{3}$ Clinical Chemistry; ${ }^{4}$ Pathology, ${ }^{5}$ Rheumatology, Leiden University Medical Center, Leiden, Netherlands

Background: In systemic lupus erythematosus (SLE) patients, excessive formation of neutrophil extracellular traps (NETs) is observed while their degradation is impaired. In vitro, immune complexes (ICX) trigger NET formation while NETderived DNA is a postulated autoantigen for anti-nuclear autoantibodies (ANAs), found in SLE. Based on these self-perpetuating mechanisms in SLE, we hypothesised that interfering with ICX formation should regress NET formation and potentially ameliorate disease.

Objectives: Investigate the effect of Rituximab+Belimumab therapy on pathogenic autoantibodies in relation to NET formation in severe refractory SLE

Methods: A phase 2A, open-label, single arm proof-of-concept study was performed wherein 16 SLE patients with severe, refractory disease were treated with a combination of CD20-mediated B-cell depletion with rituximab and sustained inhibition of B-cell activating factor with belimumab. Besides safety, the study's endpoints were chosen to address the concept of autoantibodies in relation to excessive NET formation.

Results: We demonstrated that SLE-derived immobilised IgG, but not soluble IgG, induced excessive NET formation, confirming ex vivo that ICx mediate excessive NET formation in SLE. We showed that therapeutic intervention with RTX+BLM led to specific reductions in ANAs and regression of excessive NET formation. RTX+BLM appeared to be safe and achieved clinically significant responses: low lupus disease activity state was achieved in 10 patients, renal responses in 11 patients and concomitant immunosuppressive medication was tapered in 14 out of the 16 patients.

\section{A. Effect of RTX+BLM on (auto-)antibody levels}

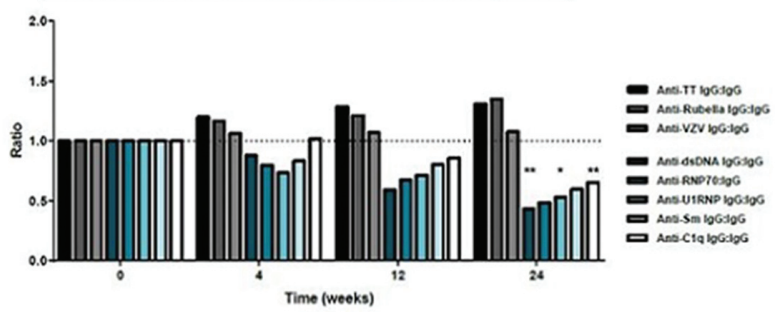

B. Effect of RTX+BLM on excessive NET formation

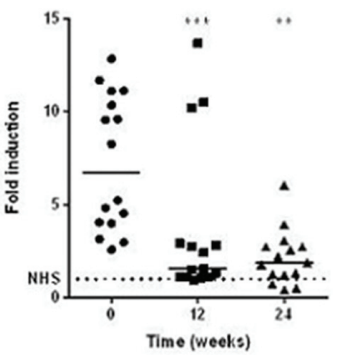

Conclusions: This study provides novel insights into clinical beneficence of reducing excessive NET formation in SLE by therapeutic targeting ANA production with RTX+BLM. Altogether putting forward a new treatment concept that specifically ameliorates underlying SLE pathophysiology.

Acknowledgements: We thank professor C. Pusey (Imperial College London, UK) for kindly providing the Syk inhibitor R406 for our experiments.

Disclosure of Interest: O. Teng Grant/research support from: GlaxoSmithKline, Consultant for: Aurinia Pharmaceuticals, GlaxoSmithKline, T. Kraaij: None declared, S. Kamerling: None declared, E. de Rooy: None declared, P. van Daele: None declared, O. Bredewold: None declared, J. Bakker: None declared, I. Bajema: None declared, H. Scherer: None declared, R. Toes: None declared, T. Huizinga: None declared, T. Rabelink: None declared, C. van Kooten: None declared

DOI: 10.1136/annrheumdis-2018-eular.6415

\section{FRI0330 \\ RITUXIMAB THERAPY IN SLE: EARLY RETREATMENT IS ASSOCIATED WITH LOWER DISEASE ACTIVITY AND A REDUCTION IN CORTICOSTEROID USE}

R.L. Batten, S. Batool, C.E. Kidd, A. Lorenzi, J. Vila, K. Walker, B. Griffiths. Rheumatology, Newcastle upon Tyne Hospitals NHS Foundation Trust, Newcastle, UK

Background: The recently published UK guidelines for the management of SLE recommend biologic therapy for severe or refractory disease. The British Isles Lupus Assessment Group Biologics Register (BILAG-BR) has shown rituximab (RTX) to be safe, effective and corticosteroid [CS] sparing when used to treat refractory SLE. In 2013 NHS England published an interim clinical commissioning policy statement with criteria determining when RTX can be used to treat SLE. Objectives: We evaluated our centre's RTX retreatment strategy in patients with SLE and the consequent outcomes (disease activity and CS dose).

Methods: Records for the first 50 patients receiving RTX for refractory SLE who consented to join BILAG-BR from our centre between December 2013 and January 2016 were analysed (data cut off July 2016). Demographics, disease activity ${ }^{\mathrm{B}-}$ LAG ${ }^{2004}$ /SLEDAI-2K), change in CS dose, retreatment schedules and adverse events were analysed.

Results: Median(IQR) age and disease duration were 42.8 (33-53) years and 9.5 (4-15.8) years respectively. Male: female ratio was $1: 25$. $80 \%$ were Caucasian, $6 \%$ Asian, $4 \%$ Caribbean and $10 \%$ other. All patients met SLICC/ACR classification criteria for SLE. The median(IQR) SLEDAI-2K scores and BILAG 2004 scores reduced from $6(4-8)$ to $4(0-4)(p<0.00001)$ and $20(10-24.5)$ to $9(2-$ 15.5) $(p<0.001)$ respectively at 6 months. Complete response was achieved in $62.8 \%$ patients (defined as loss of all BILAG $A$ and $B$ scores to $\leq 1 B$ score with no new $A / B$ scores in other organ domains). $66 \%$ patients lost all $A$ scores at 6 months. Median(IQR) daily CS dose reduced from $10 \mathrm{mg}(0-20)$ to $5 \mathrm{mg}(0.5-9.5$ at 6 months $(P=<0.001)$ and was $5 \mathrm{mg}(0-6.63)$ at last reported visit (median (IQR) 13 (12-19.5) months). 16 patients did not fully respond to baseline treatment but 11 responded to retreatment. Serious infections (requiring hospital admission) occurred in 6 patients (12\%).

30/50 patients received their 1 st course of RTX at BILAG-BR baseline visit. 23 met criteria for active disease (at least $1 \mathrm{~A}$ or 2Bs), 6 were taking an unacceptably high maintenance CS dose, and 1 was planning pregnancy. Median(IQR) CS dose in this group at baseline was $10 \mathrm{mg}(1.5-20)$, and $5 \mathrm{mg}(3-9)$ at 6 months. $70.8 \%$ demonstrated complete response at 6 months. $17(57 \%)$ went on to have retreatment due to active disease, of which $11(64.7 \%)$ had responded at 6 months post retreatment. Median(IQR) time to retreatment was $8(6-12)$ months. 20/50 patients had received retreatment at predetermined intervals prior to their baseline BILAG-BR visit. Median(IQR) numbers of previous RTX courses were 3 (2-6). Median(IQR) CS dose was $8.75 \mathrm{mg}(0-11.3)$ at baseline BILAG-BR visit and $5.5 \mathrm{mg}(0-10)$ at 6 months. Median(IQR) time from baseline to retreatment was 6 (6-9.5) months. Median(IQR) sustained response was 18 (13.5-18) months.

Percentage change $(\%)$ in steroid dose at baseline and 6 months

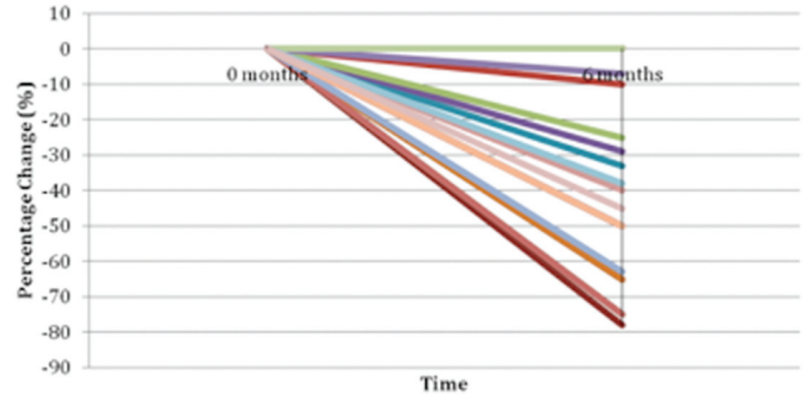

Conclusions: Historically, our centre used time from 1 st treatment to flare as a guide to a patient's future RTX retreatment schedule; patients were, on average, treated 2 months earlier than those treated under the current commissioning policy. Findings suggest that earlier retreatment led to sustained disease control and reduction of CS dose with no increase in adverse events. 11/16 incomplete res ponders responded following retreatment. Early retreatment may be associated with better outcomes for the patient and further research is needed in this area.

Disclosure of Interest: None declared

DOI: 10.1136/annrheumdis-2018-eular.4310 